PoS $\quad \begin{aligned} & \text { PROCEEDINGS } \\ & \text { OF SCIENCE }\end{aligned}$

\title{
WG2 Highlights: Small-x, Diffraction and Vector Mesons
}

\section{Robert Ciesielski}

The Rockefeller University, 1230 York Avenue, New York, NY 10065, USA

E-mail: robert.ciesielski@rockefeller.edu

\section{Yuri V. Kovchegov}

Department of Physics, The Ohio State University

Columbus, OH 43210, USA

E-mail: kovchegov.1@osu.edu

\section{Eugenio Scapparone}

INFN-Bologna, Via Irnerio 46, 40126 Bologna, Italy

E-mail: eugenio.scapparone@bo.infn. it

We summarize the results presented in the Small-x, Diffraction and Vector-Mesons Working Group at the DIS 2015 workshop.

XXIII International Workshop on Deep-Inelastic Scattering

27 April - May 12015

Dallas, Texas 


\section{Introduction}

In this article we summarize the results presented in the Small-x, Diffraction and VectorMesons Working Group at the DIS 2015 workshop. First we present the experimental overview, followed by the summary of theoretical results.

\section{Experimental overview}

\subsection{Total, elastic and soft diffractive cross sections}

The total hadronic, elastic and diffractive cross sections represent fundamental quantities of strong interactions, but cannot be calculated within the perturbative quantum chromodynamics (pQCD). Measurement of these cross sections are necessary to test and tune phenomenological QCD models, and establish a link between the non-perturbative and perturbative QCD. Several presentations focused on the new measurements from the proton-proton collisions at the LHC.

L. Adamczyk presented [1] the ATLAS measurement of the elastic and total hadronic pp cross section at $\sqrt{s}=7 \mathrm{TeV}$ [2] based on the low-pileup data taken in a special run with $\beta^{*}=90 \mathrm{~m}$ beam optics. About one million events in the region $0.0025<-t<0.38 \mathrm{GeV}^{2}$ were used to measure elastic cross section, with protons tagged in the ALFA detectors, consisting of tracking stations located at the at $z= \pm 240 \mathrm{~m}$ downstream the ATLAS detector. The total pp cross sections was obtained from the elastic scattering using the optical theorem. The cross sections $\sigma_{e l}=24.0 \pm 0.6$ $\mathrm{mb}, \sigma_{\text {tot }}=95.4 \pm 1.3 \mathrm{mb}$ have been measured (Fig. 1 left) and $\sigma_{\text {inel }}=\sigma_{\text {tot }}-\sigma_{e l}=71.3 \pm 0.9 \mathrm{mb}$ has been obtained, in agreement within errors with the TOTEM results $[3,4,5]$ of e.g. $\sigma_{e l}=25.4 \pm 1.1$ $\mathrm{mb}, \sigma_{\text {tot }}=98.6 \pm 2.2 \mathrm{mb}$ and $\sigma_{\text {inel }}=73.2 \pm 1.6 \mathrm{mb}$ (luminosity dependent method [3]).

The new TOTEM measurements of elastic proton-proton scattering at $\sqrt{s}=8 \mathrm{TeV}$ were presented by M. Deile [6]. The high-statistics data sample of about seven million events, obtained with the $\beta^{*}=90 \mathrm{~m}$ optics, was used to measure the differential cross-section in the range $0.027<|t|<0.2 \mathrm{GeV}^{2}$ [7]. With an unprecedented precision below $1 \%$ an evidence for a nonexponential differential cross-section was observed, and a purely exponential dependence was excluded with a significance greater than 7 standard deviations. Two extended parameterizations, with
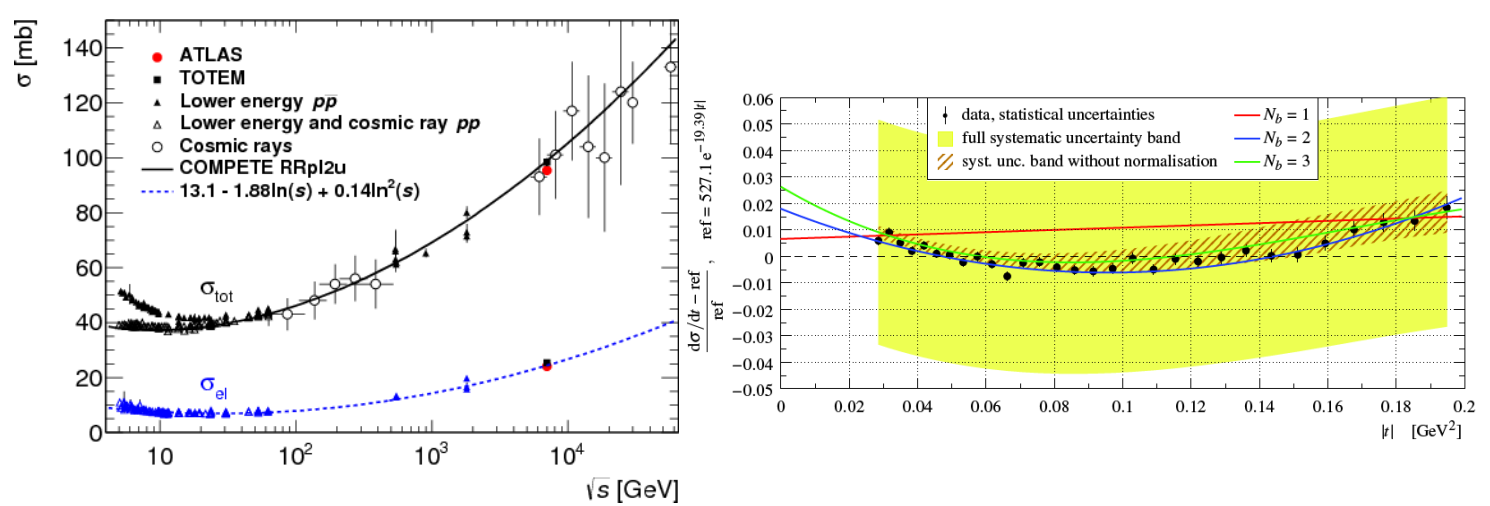

Figure 1: Left: Total and elastic pp cross section measured by ATLAS, compared to the TOTEM measurements and results from other experiments. Right: Relative difference between the $d \sigma / d|t|$ measured by TOTEM and a reference exponential $\left(527.1 \cdot e^{-19.39 \cdot|t|}\right)$; solid curves correspond to the cross section parameterization with a linear $\left(N_{b}=1\right)$, quadratic $\left(N_{b}=2\right)$ and cubic $\left(N_{b}=3\right)$ polynomials in the exponent. 
quadratic and cubic polynomials in the exponent, were found to be well compatible with the data (Fig. 1 right). Total cross sections measured by extrapolating these parameterizations to $t=0$, and further applying the optical theorem, agree with the previous TOTEM result of $\sigma_{t o t}=101.7 \pm 2.9$ $\mathrm{mb}$ [8]. Another study, currently in progress, is based on the data taken with the $\beta^{*}=1000 \mathrm{~m}$ optics, which allows to reach the $|t|$ values as low as $|t| \simeq 6 \times 10^{-4} \mathrm{GeV}^{2}$ and to observe the effects of Coulomb-nuclear interference. A study of the phase of the hadronic amplitude is ongoing.

Predictions of the total, elastic and diffractive cross sections based on the phenomenological RENORM /MBR model [9] were discussed by K. Goulianos [10]. The predictions at $\sqrt{s}=7 \mathrm{TeV}$ and $8 \mathrm{TeV}$ are in a good agreement with the ATLAS and TOTEM results. The predictions for $\sigma_{t o t}$, $\sigma_{e l}$ and $\sigma_{\text {inel }}$ at $13 \mathrm{TeV}$ are 108, 32 and $77 \mathrm{mb}$, respectively, with uncertainties of $\sim 11 \%$, which can be further reduced by a factor of $\sim 4$. The diffractive cross sections in the RENORM/MBR model are found to describe well the CDF and LHC diffractive data (see below).

G. Brona presented [11] the recent CMS results on single-diffractive (SD) and double-diffractive (DD) cross sections at $\sqrt{s}=7 \mathrm{TeV}$ [12]. Diffractive events were selected based on the presence of a forward or central pseudorapidity gap in the CMS detector $(|\eta|<4.7)$, of at least 4 and 3 units in pseudorapidity, respectively. In the forward-gap sample, subsamples enhanced in SD and DD events were further selected by requiring an absence or a presence of an energy deposit in the CASTOR calorimeter (located at $-6.6<\eta<-5.2$, in the direction of the gap). This allowed to measure the differential cross sections as a function of $\xi_{X}=M_{X}^{2} / \mathrm{s}$, in the range $-5.5<\log _{10} \xi_{X}<-2.5$, for $\log _{10} M_{Y}<0.5$ (dominated by SD) and $0.5<\log _{10} M_{Y}<1.1$ (dominated by DD), where $M_{X}$ and $M_{Y}$ correspond to diffractive masses (given in $\mathrm{GeV}$ ). The cross sections were compared to MC predictions by PYTHIA8-4C, PYTHIA8 MBR and PYTHIA6Z2* (Fig. 2), and PHOJET, QGSJET-II 03, QGSJET-II 04, EPOS. The data are able to distinguish between different diffractive models, favoring PYTHIA8-MBR simulation. Similar conclusions can be drawn from the comparison to the differential cross section measured as a function of the width of the central pseudorapidity gap $\Delta \eta$ for $\Delta \eta>3, \log _{10} M_{X}>1.1$, and $\log _{10} M_{Y}>1.1$ (DD dominated). The cross sections integrated over the above three regions were used to extract total SD and DD cross sections of $\sigma^{\mathrm{SD}}=8.84 \pm 0.08(\mathrm{stat})_{-1.38}^{+1.49}(\mathrm{syst})_{-0.37}^{+1.17}$ (extrap) mb and $\sigma^{\mathrm{DD}}=5.17 \pm 0.08(\mathrm{stat})_{-0.57}^{+0.55}$ (syst) ${ }_{-0.51}^{+1.62}$ (extrap) $\mathrm{mb}$ in the regions of $\xi<0.05$ and $\Delta \eta>3$, respectively (after an extrapolation by a factor of $\sim 2$ with PYTHIA8-MBR).
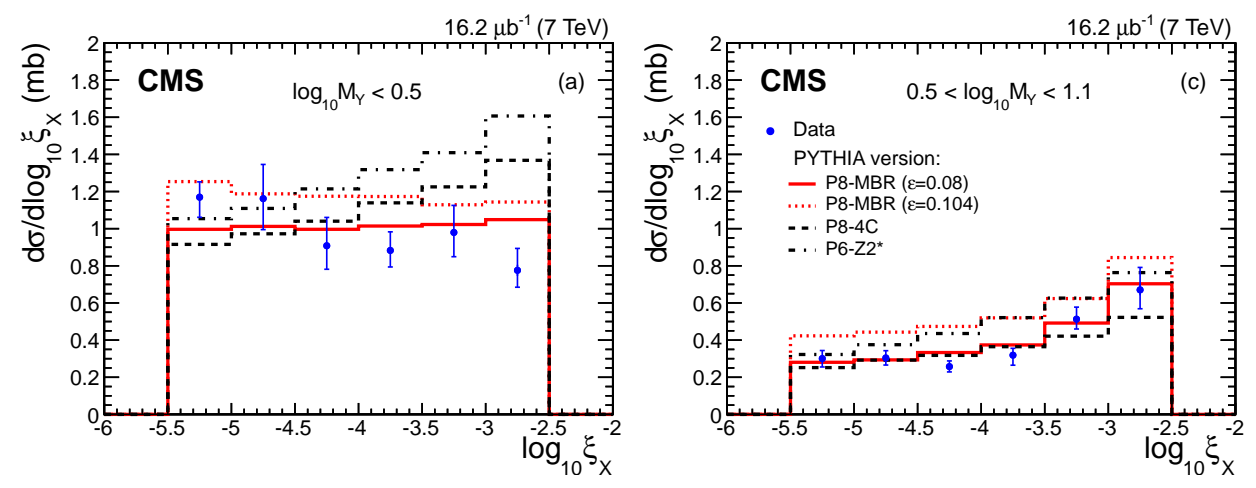

Figure 2: Cross sections $d \sigma / d \log _{10} \xi_{X}$ for (left) $\log _{10} M_{Y}<0.5$ (SD dominated) and (right) $0.5<$ $\log _{10} M_{Y}<1.1$ (DD dominated), compared to PYTHIA8-4C, PYTHIA8 MBR, PYTHIA6-Z2* predictions. 


\subsection{Hard diffraction}

If a hard scale is present in a diffractive process, typically provided by high-mass or high- $p_{T}$ objects in the final state, the corresponding cross section can be calculated within pQCD. Calculations can be factorized into process-dependent partonic cross sections and process-independent diffractive parton distribution functions (DPDFs), which have to be determined experimentally. The universality of this approach, however, is limited by additional soft multiparton interactions, which may spoil the diffractive gap. Factorization breaking has been observed in the diffractive dijet production at Tevatron, for which predictions based on HERA DPDFs overestimate the measurements by about one order of magnitude.

The new H1 measurement of diffractive dijet production in DIS [13] was presented by S. Schmitt [14]. Diffractive dijet events were selected by means of the LRG method. The measured cross sections are well described in the shapes and normalization (Fig. 3 left) by next-to-leading order (NLO) pQCD predictions evaluated with input DPDFs, confirming at improved precision previous $\mathrm{H} 1$ measurements. The new data are more precise than NLO predictions, and can be used to further constrain DPDFs. S. Schmitt also presented [15] the published H1 measurement of diffractive dijet production in DIS and photoproduction $\left(Q^{2}=0\right)$ with a leading proton detected in the Very Forward Proton Spectrometer (FVPS) [16]. The proton detection in VFPS assures that the diffractive samples are free of background originating from low-mass dissociative states. Measured cross sections were compared to NLO pQCD calculations based on DPDFs (Fig. 3 right), showing that while DIS data are well described by the predictions, the NLO calculations overestimate the measured photoproduction cross section by almost a factor of two (with no clear dependence on any variable). The double ratio of photoproduction to DIS and data to NLO predictions yields a
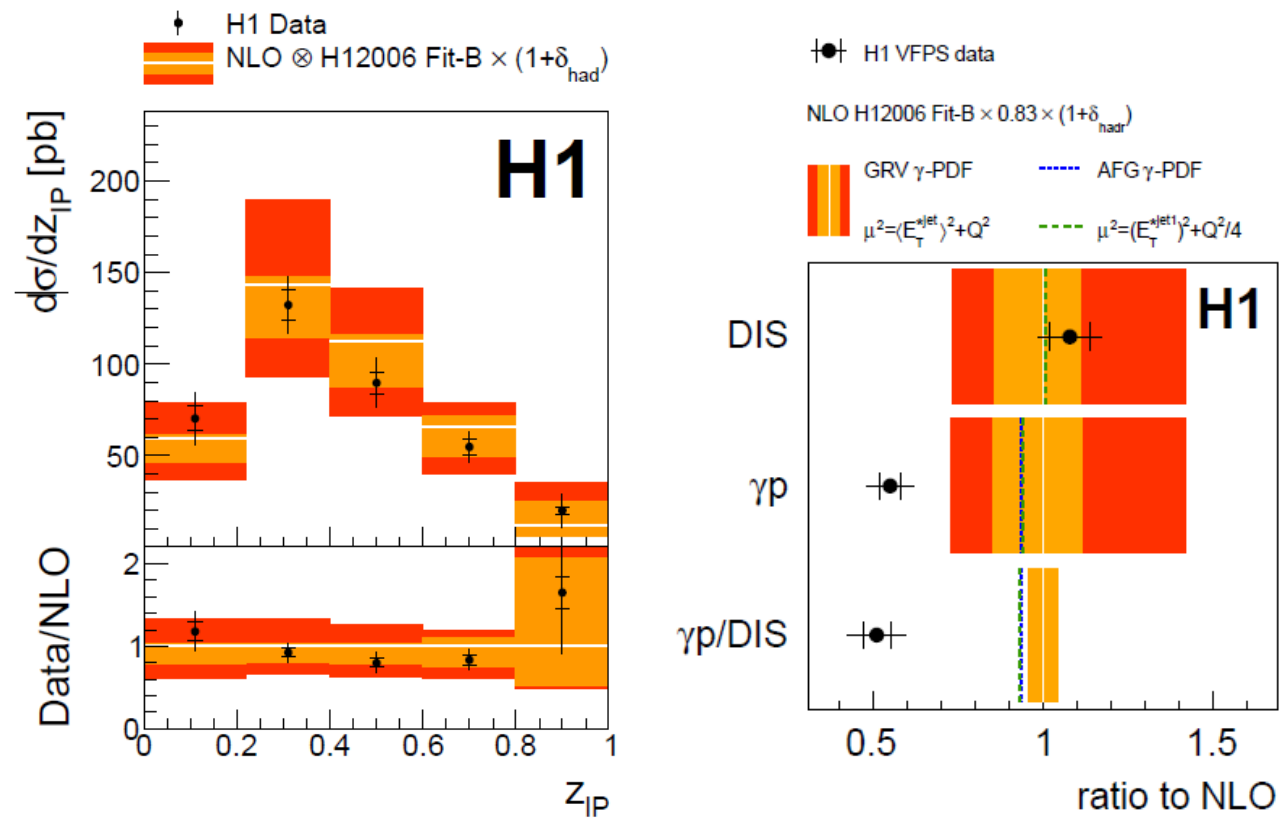

Figure 3: $\mathrm{H} 1$ measurements of (left) differential cross section as a function of $x_{I P}$ (Pomeron momentum fraction entering hard subprocess) for diffractive dijet production in DIS, compared to NLO theory predictions, and (right) diffractive dijet DIS and photoproduction cross sections, normalized to the NLO calculations. 

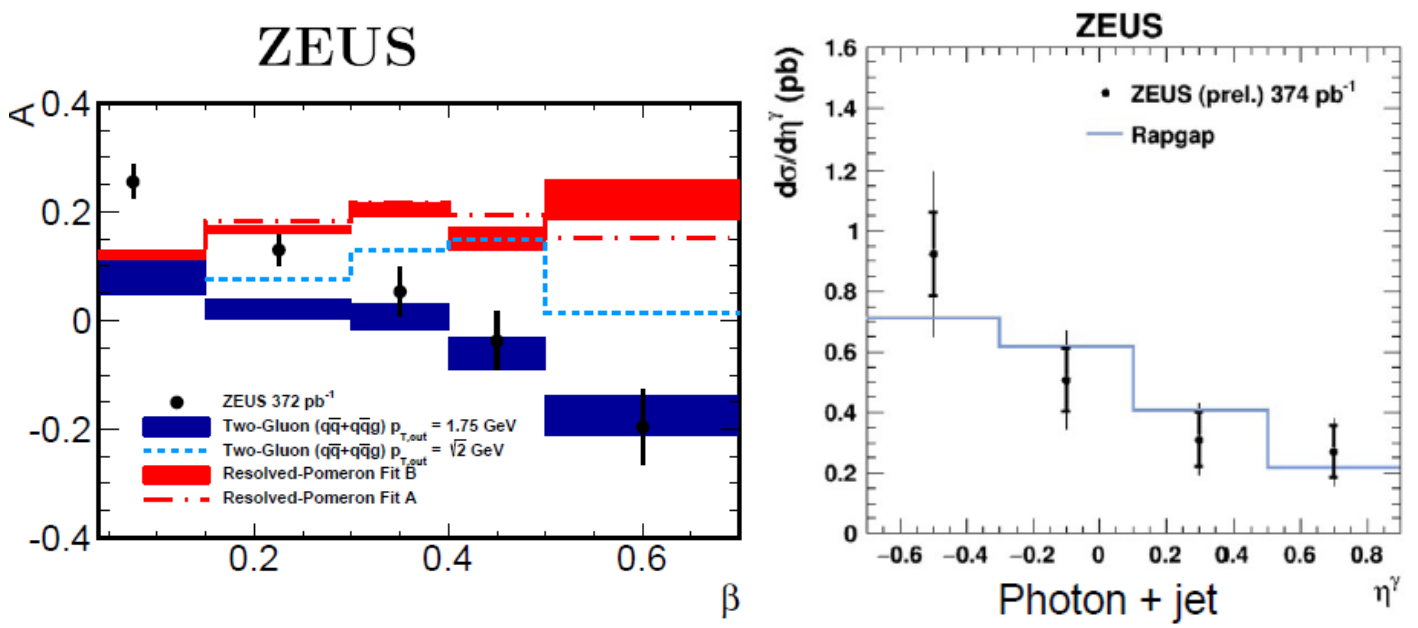

Figure 4: ZEUS measurements of (left) the shape parameter $\mathrm{A}$ as a function of $\beta$ (Pomeron momentum fraction entering hard subprocess) measured in the analysis of exclusive dijet production, compared to theory predictions, and (right) differential cross section as a function pseudorapidity of the isolated photon in the diffractive photon+jet events, compared to the RAPGAP MC simulation.

suppression factor of $0.51 \pm 0.09$, interpreted as the $\mathrm{H} 1$ observation of factorization breaking in diffractive photoproduction of dijets.

The ZEUS measurement of exclusive dijets in diffractive DIS [17] was presented by L. Adamczyk [18]. The cross section were measured as a function of $\beta$ (Pomeron momentum fraction entering hard subprocess) and azimuthal angle $\varphi$ (defined by the $\gamma^{*}$-dijet and $\gamma^{*}$-e planes in the rest frame of the diffractive final state). The $\varphi$ cross sections, measured in five bins of $\beta$, were fitted with a form $1+A \cos (2 \varphi)$, with the shape parameter $A$ carrying information about the nature of the production mechanism. Fig. 4 presents the A vs. $\beta$ dependence compared to theory predictions, the data favor the two-gluon-exchange model (pQCD calculations using proton PDFs) over the resolved-Pomeron model (boson-gluon fusion with Pomeron PDFs).

P. Bussey summarized the first ZEUS measurement of diffractive photoproduction of isolated photons [19]. The process, while rare, is interesting as it tests the presence of a quark content in the Pomeron. Cross section has been measured for diffractive events with an isolated photon, inclusively and with an accompanying jet, as a function of photon and jet $E_{T}$ and $\eta$, and diffractive variable $z_{I P}$. The cross section for photons with a jet was found to be a high fraction of the inclusive photon cross section. The results were compared to RAPGAP predictions based on H1 DPDFs. The data show a peak at high values of $z_{I P}$, which is not well described by the simulation. In all other cases, RAPGAP describes the data well (see e.g. the $\eta^{\gamma}$ distribution in Fig. 4, right).

Two talks addressed the plans for measurements of hard diffractive processes with joint CMS and TOTEM detectors. M. Deile [6] briefly reminded the special low-pileup CMS and TOTEM run taken in 2012 with the $\beta^{*}=90 \mathrm{~m}$ optics. Although statistically limited, the data were used to perform the first studies of events with diffractive final state reconstructed in the CMS detector and scattered proton(s) in the TOTEM Roman Pots. A similar low-pileup run at $\sqrt{s}=13 \mathrm{TeV}$ is scheduled for the Fall 2015, to study processes such as e.g. single- and central-diffractive dijet production or low-mass central diffractive spectroscopy, including a search for glueballs. The planned installation of the diamond timing detectors will allow to collect the data at a moderate pileup in 
2016. M. Albrow presented [20] a summary of the CT-PPS project, the collaboration between CMS and TOTEM (CT) for Precision Proton Spectrometers (PPS) [21], which was recently approved by the LHCC and the CERN Research Board. The CT-PPS consists of 3D silicon trackers and Cherenkov timing detectors, situated in the TOTEM Roman pots. A cylindrical design and an excellent time resolution of about 20 ps will allow operation during standard high-luminosity and high-pileup runs, to study $p+p \rightarrow p+X+p$ events, with $M(X)>300 \mathrm{GeV}$, including the Pomeron-Pomeron induced central (exclusive) production of dijets or photon-photon production of $W W$ pairs. The detectors are planned for installation in January 2016, aiming for physics in 2016. A similar project, AFP (ATLAS Forward Physics), was briefly introduced by L. Adamczyk [1].

\subsection{Exclusive production}

The central exclusive production in the hadron-hadron collisions proceeds by the photonphoton, Pomeron-Pomeron, or photon-Pomeron exchanges. The $\gamma-\gamma$ interactions allow to test the QED predictions; the IP-IP exchange selects $I^{G}\left(J^{P C}\right)=0^{+}(\text {even })^{++}$states and allows to study isoscalar meson spectroscopy, e.g. production of glueballs; the $\gamma$-IP process, with vector mesons in the final state, allows to test pQCD description of the color-singlet exchange. The theory has been established based on extensive studies of vector meson production at HERA. The process has been shown to exhibit high sensitivity to gluon distributions in the proton, at the LHC it may be used to search for effects such as gluon saturation.

There was one talk at DIS2015 with vector meson results from HERA. M. Wing and N. Kovalchuk presented the ZEUS measurement of the ratio of cross sections for the exclusive production of $\psi^{\prime}$ and $J / \psi$ mesons [22]. Fig. 5 (left) presents the $\sigma_{\psi^{\prime}} / \sigma_{J / \psi}$ ratio measured as a function of photon virtuality $Q^{2}$, showing that the $\psi^{\prime}$ cross section is suppressed w.r.t. $J / \psi$ cross section, predominantly due to a difference in the vector meson wave-functions, i.e. a node in the wave-function of the $\psi^{\prime}$. The cross section ratio rises with $Q^{2}$, which is reproduced by theoretical predictions.

M. Albrow presented [23] the recent CDF measurement of the central exclusive $\pi^{+} \pi^{-}$production in proton-antiproton collisions at $\sqrt{s}=0.9$ and $1.96 \mathrm{TeV}$ [24], dominated by the PomeronPomeron exchange. The invariant mass spectrum for events with two oppositely charged particles

\section{ZEUS}

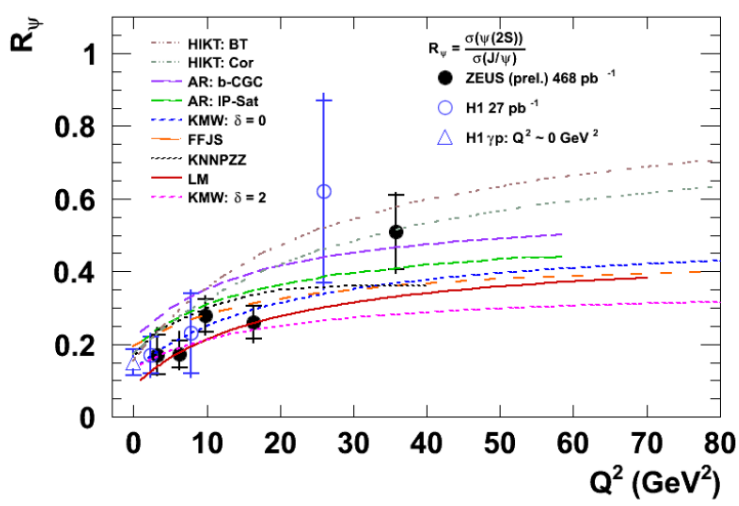

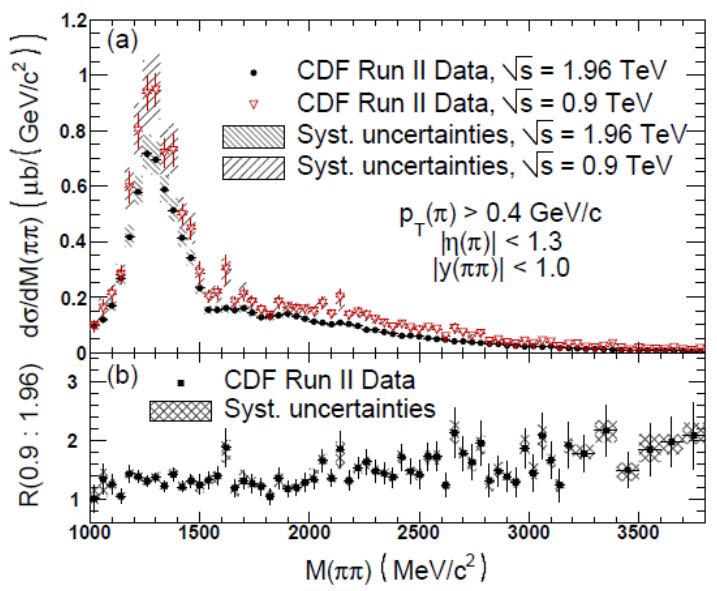

Figure 5: Left: Ratio of $\psi^{\prime}$ to $J / \psi$ cross sections measured as a function of $Q^{2}$ by ZEUS. Right: Cross section as a function of the $\pi^{+} \pi^{-}$invariant mass measured at $\sqrt{s}=0.9 \mathrm{TeV}$ and $1.96 \mathrm{TeV}$ by CDF. 

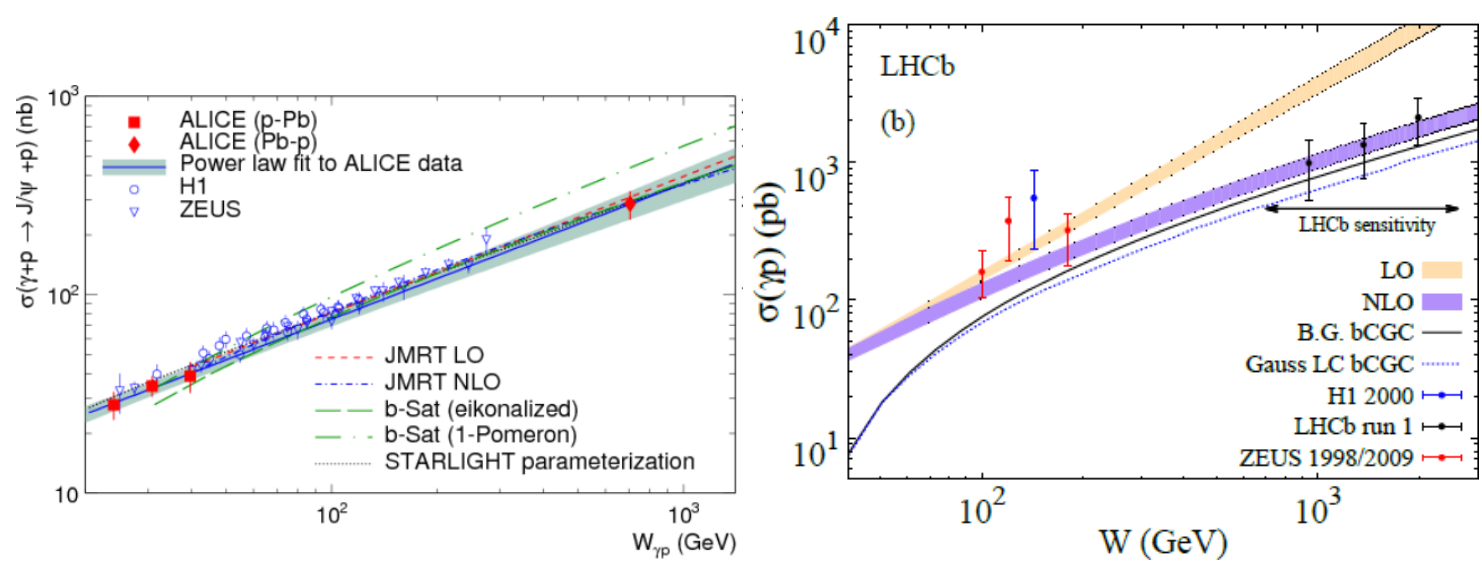

Figure 6: Cross sections of exclusive vector-meson production measured as a function of center-of-mass energy $W_{\gamma p}$ for the (left) $J / \psi$ meson by ALICE and (right) $\Upsilon$ meson by LHCb.

(assumed to be pions) and no other particles detected in $|\eta|<5.9$, is presented in Fig. 5 (right). It shows a clear structure of isoscalar meson resonances, attributed to $f_{0}$ and $f_{0}(1270)$, and some higher mass structure which cannot be clearly assigned to know states. More statistics is needed to make firm conclusions about observed states. The ratio of the cross sections at the two energies $R(0.9: 1.96)=1.3$ agrees with the Regge phenomenology expectations of $\sim 1 / \ln (s)$ dependence.

The results on central exclusive production at the LHC were discussed by R. Wallace for LHCb [25], J. Adam for ALICE [26], and by M. Murray for CMS [27] experiments.

LHCb measured cross sections for the exclusive production of $J / \psi$ and $\psi^{\prime}$ mesons at $\sqrt{s}=7$ $\mathrm{TeV}$ [28], based on the sample of dimuons with forward rapidity $2<y<4.5$. The separation of the exclusive events from those in which proton dissociates was done by means of a fit to the dimuon $p_{T}$ spectrum with two exponential functions. Exclusive events were used to measure the cross section as a function of dimuon rapidity, which agrees with several theoretical models. The LHCb data favors NLO over LO QCD predictions, and agree with the models that include saturation effects. The $J / \psi$ rapidity cross section was also used to extract the cross section as a function of the photonproton center-of-mass energy $W_{\gamma p}$. In the high- $W_{\gamma p}$ region $\left(400<W_{\gamma p}<1100\right)$ the data are well described by the function $W^{\delta}, \delta=0.67$, extracted from the fit to the HERA data $\left(30<W_{\gamma p}<300\right)$. LHCb has also presented a new measurement of the cross section for the exclusive $\Upsilon$ production at $\sqrt{s}=7$ and $8 \mathrm{TeV}$ [29]. The cross section was measured as a function of dimuon rapidity and $W_{\gamma p}$, after subtracting the proton-dissociative events and $\Upsilon$ events from the $\chi_{b}$ meson decays. The results were compared to the LO and NLO QCD predictions, favoring strongly the NLO calculations (Fig. 6 right). The first measurement of the $I P$-IP induced production of $J / \psi+J / \psi$ and $J / \psi+\psi^{\prime}$ pairs in proton-proton collisions [30] was also discussed. $57 \mathrm{~J} / \psi+\mathrm{J} / \psi$ candidates and $7 \mathrm{~J} / \psi+\psi^{\prime}$ have been found in events with four tracks and at least 3 muons. The cross section for the exclusive $J / \psi+J / \psi$ production was measured to be $24 \pm 9 \mathrm{pb}$, in agreement with theoretical predictions. For the future analyzes, the installation of HERSCHEL shower counters down to $\eta= \pm 9$ will greatly reduce the contribution from non-exclusive background.

ALICE measured the exclusive $J / \psi$ production in p-Pb collision, for the $J / \psi$ with forward rapidity $2.5<y<4$ [31]. While in pp photoproduction the analysis suffers from the intrinsic impossibility to identify the photon emitter and the photon target (reflected in an ambiguity in $W_{\gamma p}$ 
determination), in $\mathrm{p}-\mathrm{Pb}$ collisions the lead-ion predominantly serves as the source of the photon. Exclusive events were selected after subtracting the non-exclusive contribution, which was estimated from the data using events with increased energy deposition in the direction of proton beam. The photon-proton cross section measured as a function of $W_{\gamma p}$ is shown in Fig. 6 (left), compared to HERA results. The parameters of the power-law fit to ALICE results are consistent with those of HERA, indicating no change in gluon behavior at the LHC energies compared to HERA. The cross section of coherent $J / \psi$ photoproduction was also measured in $\mathrm{Pb}-\mathrm{Pb}$ collisions, in two rapidity intervals by ALICE $[32,33]$ and in one rapidity interval by CMS [34]. This process, with the lead-ion serving as both a source and a target, allows a study of nuclear gluon shadowing. The ALICE and CMS data (Fig. 7) agree with the models which include nuclear gluon shadowing employing the EPS09 parametrization. ALICE also measured photoproduction of $\rho^{0}$ in $\mathrm{Pb}-\mathrm{Pb}$

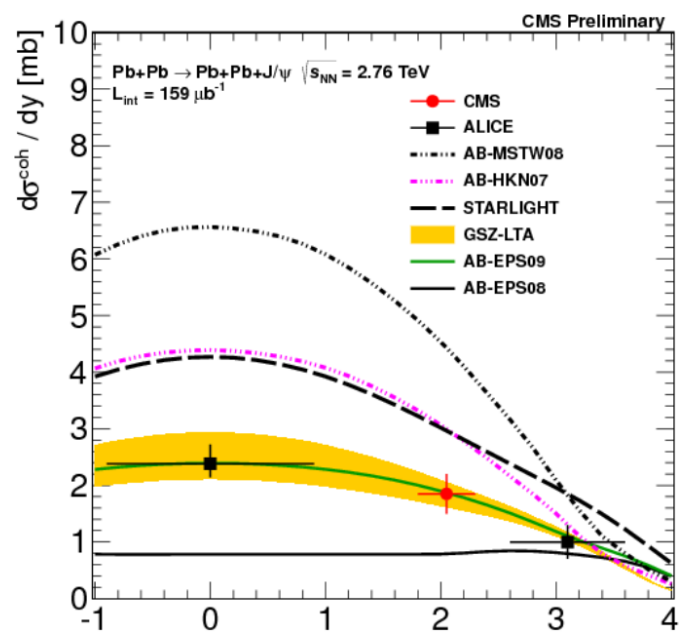

Figure 7: Exclusive $J / \psi$ production cross section measured in $\mathrm{Pb}-\mathrm{Pb}$ collisions at $\sqrt{s_{N N}}=2.76 \mathrm{TeV}$ by ALICE and CMS. collisions [35]. The $\rho^{0}$ cross section is well described by the STARLIGHT simulation using the Glauber model and the calculation by Goncalves and Machado, based on the Color Dipole model.

Furthermore, J. Nystrand summarized exclusive and inclusive photonuclear interactions at RHIC and LHC, and comparison to MC simulations [36]. M. Ducati [37] and A. Rezaeian [38] presented the comparison of the predictions of their models to the central exclusive data.

\subsection{The Electron Ion Collider (EIC)}

The US Nuclear Science Advisory Committee (NSAC) was recently requested by the DOE/NSF to conduct a new study on priorities and recommendations for the future US nuclear physics research. The NSAC produced a 2015 Long Range Plan, released in the Fall of 2015: one of the most important outcomes was the recommendation for building a high energy high-luminosity polarized Electron Ion Collider (EIC). The EIC is designated as the highest priority for the new facility construction following the completion of FRIB. Physics potential of this future collider was discussed at DIS2015 in several sessions: in the WG2 parallel session T. Ullrich presented a review of the capabilities of the Sartre code [39], providing useful predictions for the observables to be studied at EIC [40]. Diffractive production of light vector mesons, such as $\rho$ and $\phi$ mesons, at $1<Q^{2}<10$ $\mathrm{GeV}^{2} / \mathrm{c}^{2}$ is a powerful tool to study the gluon distribution of heavy nuclei. In addition, the pattern of the $d \sigma / d t$ distribution as a function of $t$ is reported to be sensitive to the onset of saturation effects in the dipole-nucleus scattering: two different models included in Sartre, one including saturation effects and another one without saturation effects (labeled b-Sat and b-NonSat respectively), give appreciable differences. A good $t$ resolution and high luminosity are required to perform an effective study. Similar conclusions were presented in the talk of A. Rezaeian [38], based on the impact-parameter dependent Color Glass Condensate (b-CGC) and Saturation (IP-Sat) dipole models. 


\subsection{Neutrino-Nucleus interactions}

Neutrino physics represents an interesting area of study, in which electro-weak and nuclear processes interfere. In order to correctly interpret the oscillation data one needs to understand the nuclear effect in the $v$-nucleus interactions, such as e.g. meson exchange currents or short-range nucleon-nucleon correlations, etc.

X. Tian presented measurements of coherent production of $\rho^{+} / \rho^{0}$ mesons in $v$-Carbon interactions with the NOMAD detector [41]. The coherent $\rho^{+}$results are the most precise measurement up to date, described well by theoretical models based on the CVC (Conservation of the Vector Current) hypothesis, with little contribution from longitudinally polarized $\rho^{+}$. The $\rho^{0}$ results represent the first observation of the neutrino-induced $\mathrm{NC}$ coherent $\rho^{0}$ production, which rate also agrees with predictions. The processes can further be studied with the DUNE/LBNF near detector.

J. Asaadi presented the measurements of the CC $v$-Ar interactions with zero pions in the final state and NC $\pi^{0}$ production using the liquid argon TPC chamber of the ArgoNeuT experiment [42]. The zero-pion events were studied as a function of the number of outgoing protons in the event, and found to be well described by the GIBUU prediction. The subsample of $\mu+2 p$ events was also used to look for hints of nucleon-nucleon short range correlations. The NC $\pi^{0}$ process, first time ever measured in the $v$-Ar interactions, agrees with the GENIE and NuWro simulations.

\section{Theory overview}

\subsection{Introduction to Saturation Physics}

The main characteristic of small- $x$ physics is a large number of quarks and gluons at small- $x$, as illustrated in Fig. 8 using the parton distribution functions (PDFs) extracted from the HERA DIS data on a proton. The gluon and sea-quark PDFs appear to rise with decreasing $x$, dominating the small- $x$ wave function of the proton. Furthermore, the contribution of gluons dominates over the sea quarks.

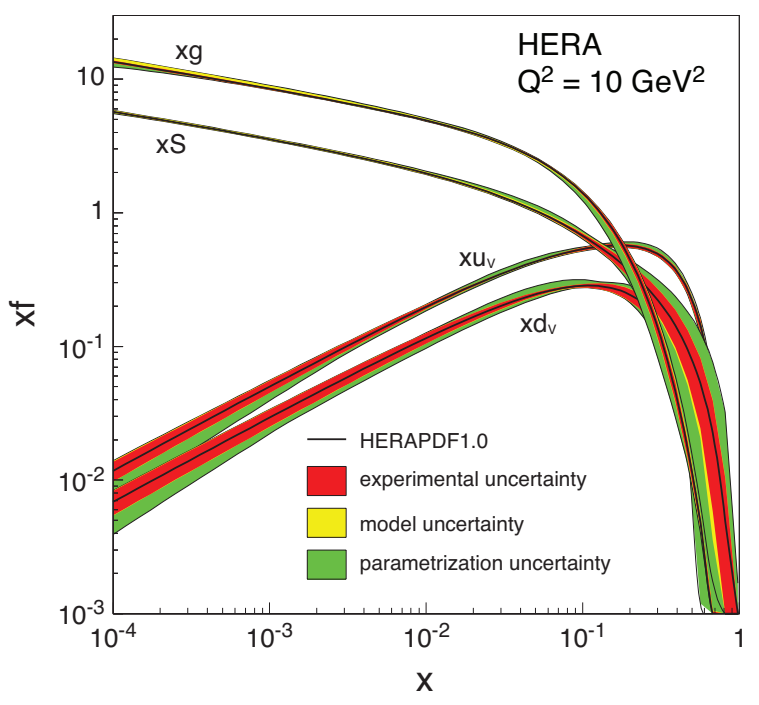

Figure 8: Quark and gluon PDFs in a proton. 
Let us imagine the spatial structure of the proton in the transverse plane, as depicted in Fig. 9. We begin in the left panel corresponding to some initial value of Bjorken- $x$, labeled $x_{0}$ : at this value of $x$ we have several partons in the proton wave function, denoted by blobs in the left panel of Fig. 9. As $x$ decreases, the number of partons increases. The new partons populate the transverse cross-section of the proton, creating a densely packed system, as shown in the right panel of Fig. 9. As more partons are produced in the QCD evolution towards low- $x$, the production of partons of a given transverse size saturates at sufficiently small $x$, since no other partons of this size can fit in the proton. Further production of smaller- $x$ partons moves to smaller transverse sizes, such that the typical newly produced partons are smaller than the ones created in the previous steps of the evolution. The typical transverse size of the partons that saturate at a given Bjorken $x$ is $1 / Q_{s}(x)$, where $Q_{s}(x)$ has the units of momentum and is called the saturation scale. Saturated small- $x$ wave function of a boosted proton or nucleus (as described within the saturation formalism) is sometimes referred to as the color glass condensate (CGC) (see the reviews [43, 44, 45, 46, 47, 48, 49, 50] and the book [51]).

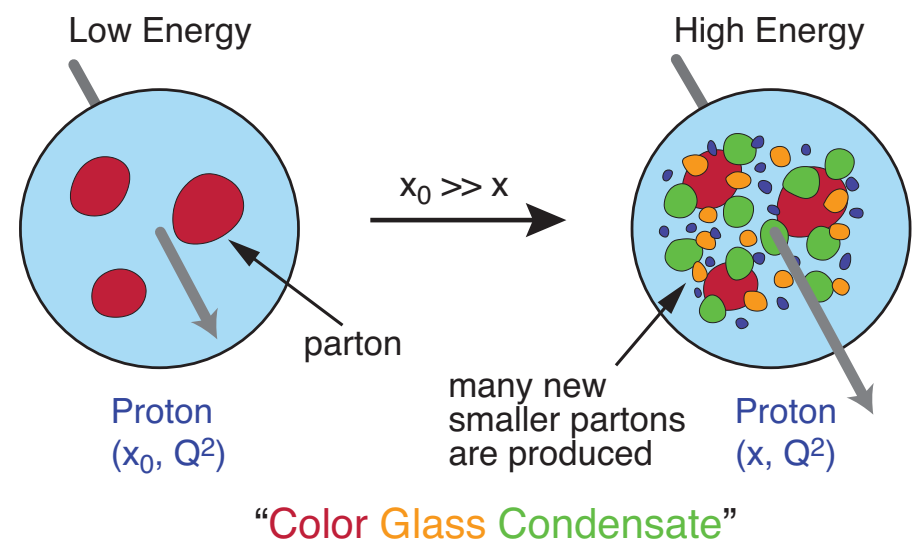

Figure 9: Quark and gluon PDFs in a proton.

Another way of thinking about saturation is as follows. Imagine the usual (linear) QCD evolution as a series of splittings, which only increase the number of partons, as shown in the first term on the right-hand side of Fig. 10. These splittings and the resulting increase in the number of small- $x$ quarks and gluons may account for the PDF growth shown in Fig. 8. However, when the density of quarks and gluons becomes large, another competing mechanism becomes important. This mechanism is recombination, and it is illustrated in the second term on the right of Fig. 10. Recombinations decrease the number of partons in the proton wave function. Once a certain critical parton density is reached, the two processes, spitting and recombination, balance each other, and the systems enters the saturation regime.

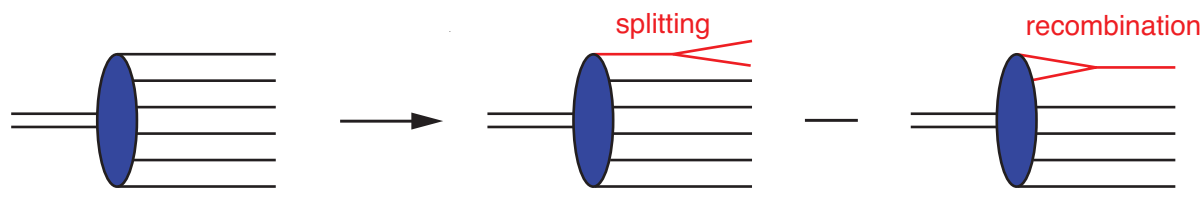

Figure 10: Pictorial representation of the nonlinear small- $x$ evolution. All lines can be thought of as gluons. 
To understand the transition to the saturation regime a little more formally, let us introduce the function counting the number of gluons with a given value of Bjorken $x$ and transverse momentum $k_{T}, N\left(x, k_{T}^{2}\right)$. At the leading logarithm- $1 / x$ level, the evolution of $N\left(x, k_{T}^{2}\right)$ towards low$x$ is schematically represented in Fig. 10. Note that the splitting contribution is proportional to $N\left(x, k_{T}^{2}\right)$, while the recombination requires two partons to participate, and is hence proportional to $\left[N\left(x, k_{T}^{2}\right)\right]^{2}$. We can therefore write the following evolution equation:

$$
\frac{\partial}{\partial \ln (1 / x)} N\left(x, k_{T}^{2}\right)=\alpha_{s} K_{B F K L} \otimes N\left(x, k_{T}^{2}\right)-\alpha_{s}\left[N\left(x, k_{T}^{2}\right)\right]^{2} .
$$

This is the large- $N_{c}$ BK evolution equation [52, 53, 54, 55], also capturing some of the dynamics of the all- $N_{c}$ JIMWLK evolution $[56,57,58,59] . K_{B F K L}$ is the kernel of the linear BFKL small- $x$ evolution equation $[60,61]$.

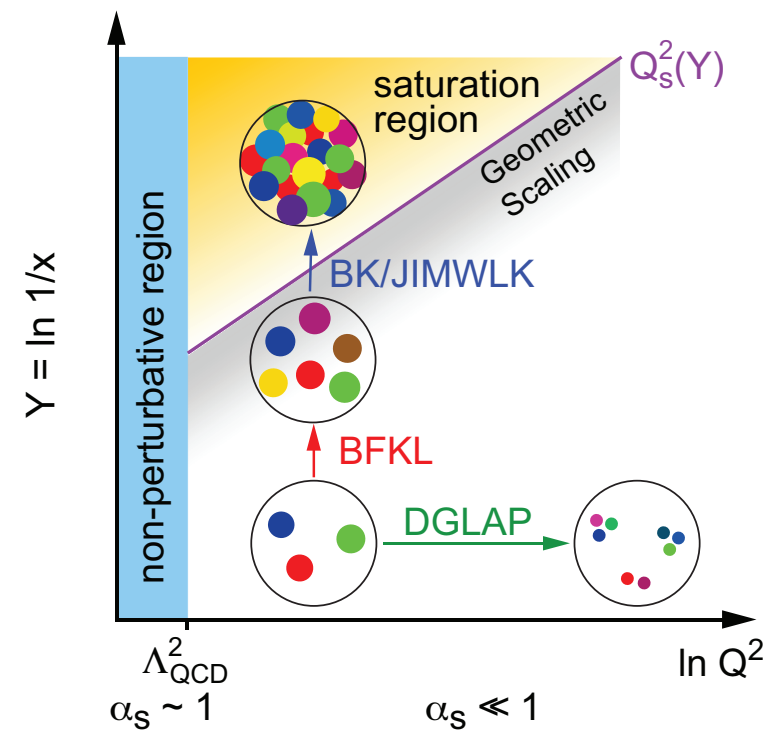

Figure 11: The map of high-energy QCD.

Our understanding of QCD evolution with emphasis on small- $x$ is summarized in the map of high-energy QCD depicted in Fig. 11. There the action of several evolution equations are shown in the $\left(x, Q^{2}\right)$ plane. The "standard" DGLAP evolution evolves PDFs toward large $Q^{2}$, as shown by the right-pointing arrow in Fig. 11. The BFKL equation evolves the unintegrated gluon distribution (gluon transverse momentum distribution (TMD)) towards small- $x$, shown by the vertical arrow. However, at very small- $x$ the BFKL evolution breaks down due to the presence of saturation effects, and the non-linear BK/JIMWLK evolution takes over. The transition to the saturation region, pictured by the gold color in the upper left corner of Fig. 11, is characterized by the saturation scale $Q_{s}^{2}(Y)$ with rapidity $Y=\ln (1 / x)$. It is important to note that $Q_{s}(Y)$ is a growing function of both rapidity $Y$ and the atomic number $A$ for a nuclear target. Therefore, for small enough $x$ and/or for large enough nuclei the saturation scale can get much larger than $\Lambda_{Q C D}$ justifying the small-coupling $\left(\alpha_{s} \ll 1\right)$ approach to the problem. 


\subsection{Geometric Scaling}

An important prediction of saturation physics is geometric scaling: the solution of the nonlinear equation (3.1) in most of the phase space region is a function of one scaling variable,

$$
N\left(x, k_{T}^{2}\right)=N\left(k_{T}^{2} / Q_{s}^{2}(x)\right),
$$

instead of being a function of $x$ and $k_{T}$ separately $[62,63]$. The argument for geometric scaling can be constructed on general grounds, by arguing that $Q_{s}(x)$ is the only relevant scale in the problem, such that each power of $k_{T}$ should come in as $k_{T} / Q_{s}(x)$ [64, 65, 66]. Geometric scaling was first observed in HERA DIS data [67].

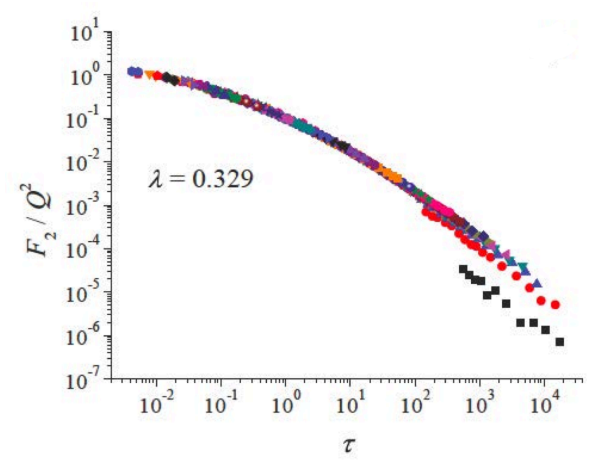

Figure 12: Geometric scaling in DIS. Here $\tau=Q^{2} / Q_{s}^{2}(x)$.

There was one talk in WG 2 on expanding the scope of observation of geometric scaling in the data. The work of M. Praszalowicz [68] reconfirmed the existing observation of geometric scaling in the HERA data on $F_{2}$ structure function and extended the scaling region up to fairly large $x$ of 0.08. This is illustrated in Fig. 12, where $F_{2} / Q^{2}$ is plotted as a function of the scaling variable $\tau=Q^{2} / Q_{s}^{2}(x)$.
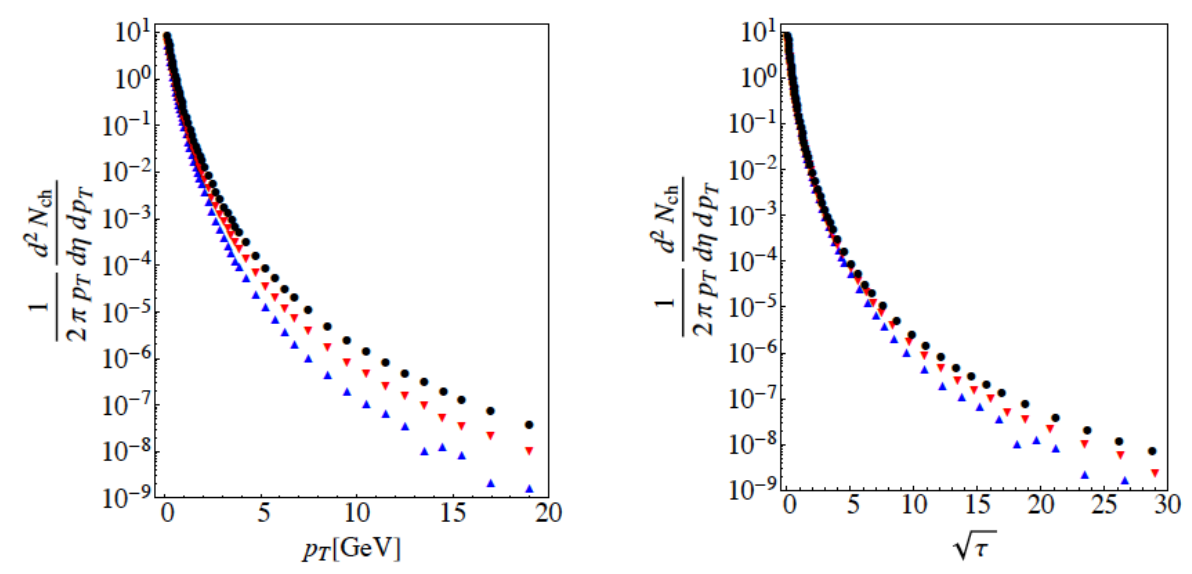

Figure 13: Geometric scaling in the particle spectra in proton-proton collisions. Here $\tau=p_{T}^{2} / Q_{s}^{2}\left(p_{T} / \sqrt{s}\right)$.

Further search for geometric scaling revealed its presence in $p+p$ and $A+A$ collisions as well. The former is illustrated in Fig. 13 with the plot obtained using ALICE data [69] for the particle 
number distribution as a function of $p_{T}$ in the left panel (for three different center-of-mass energies $s)$ and as a function of $\sqrt{\tau}=p_{T} / Q_{s}\left(p_{T} / \sqrt{s}\right)$ in the right panel. The particle spectra look distinctly different in the left panel, and move much closer together for a part of phase space depicted in the right panel, indicating that geometric scaling may be present in the particle spectra generated in $p+p$ collisions. As a word of caution we need to point out the logarithmic scale on the vertical axis in Figs. 12 and 13, which makes it harder to see the details of scaling.

The big question here is whether the geometric scaling found in Figs. 12 and 13 is, in fact, a signal of saturation, since the $F_{2}$ structure function and particle spectra can also be well-described using the physics based on DGLAP evolution. While the evidence for saturation is compelling, one has to point out that the $x$-dependence of the saturation scales used in the scaling variables of Figs. 12 and 13 is slightly different. Further, more quantitative theoretical understanding of this difference appears to be needed in order to draw a more solid conclusion.

\subsection{Small- $x$ Evolution at NLO}

Currently we know the BFKL evolution kernel up to next-to-leading order (order- $\alpha_{s}^{2}$ ) [70, 71]. Similarly the BK evolution kernel is known up to order- $\alpha_{s}^{2}$ [72]. The JIMWLK evolution kernel at NLO was presented in WG 2 by three speakers, G. Chirilli, A. Grabovsky and M. Lublinsky, representing three different calculations $[73,74,75]$. The detailed extensive expression for the kernel will not be presented in this summary, and interested readers are referred to the original papers $[73,74,75]$. We need to mention that all three calculations are in agreement with each other.

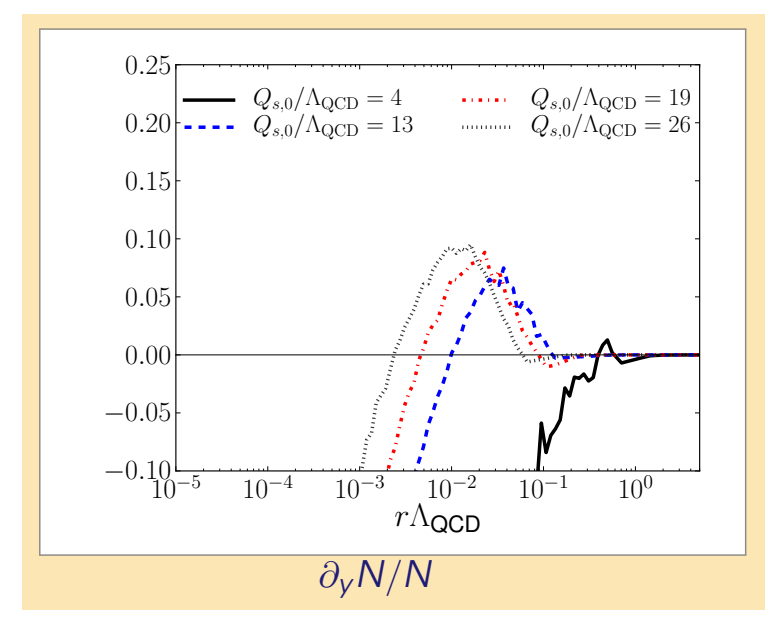

Figure 14: A plot illustrating instability of the NLO BK solution.

Since BK and JIMWLK evolution equations allow only for a numerical solution, to understand the impact of NLO corrections one has to solve the NLO BK and JIMWLK evolutions numerically. An attempt to construct a numerical solution of the NLO BK evolution was presented by T. Lappi [76]. The calculation ran into an issue which did not allow the authors to arrive at a complete numerical solution of NLO BK. At small dipole $\operatorname{sizes}^{1}$ the LO+NLO kernel acting on the right-

\footnotetext{
${ }^{1}$ The BK equation is usually written in transverse coordinate space for the color-dipole scattering amplitudes which are related to the gluon number distributions in transverse momentum space we have employed in Eq. (3.1).
} 
hand side of NLO BK equation gives very small numbers, almost indistinguishable from zero, such that fluctuations in the numerics make the right-hand side of the equation, and, therefore, the speed of the evolution $\partial_{Y} N \equiv \partial N / \partial Y$, negative. This is illustrated in Fig. 14 where $\partial_{Y} N / N$ is plotted as a function of the dipole size $r$ for several different initial values of the initial saturation scale. Negative $\partial_{Y} N$ at one step of evolution, combined with a small value of $N$ at the same step, may lead to negative forward dipole amplitude $N$ at the next step of evolution, which is unphysical, since it leads to negative dipole-target scattering cross section. It appears that a direct numerical solution of NLO BK equation is very hard if at all possible.

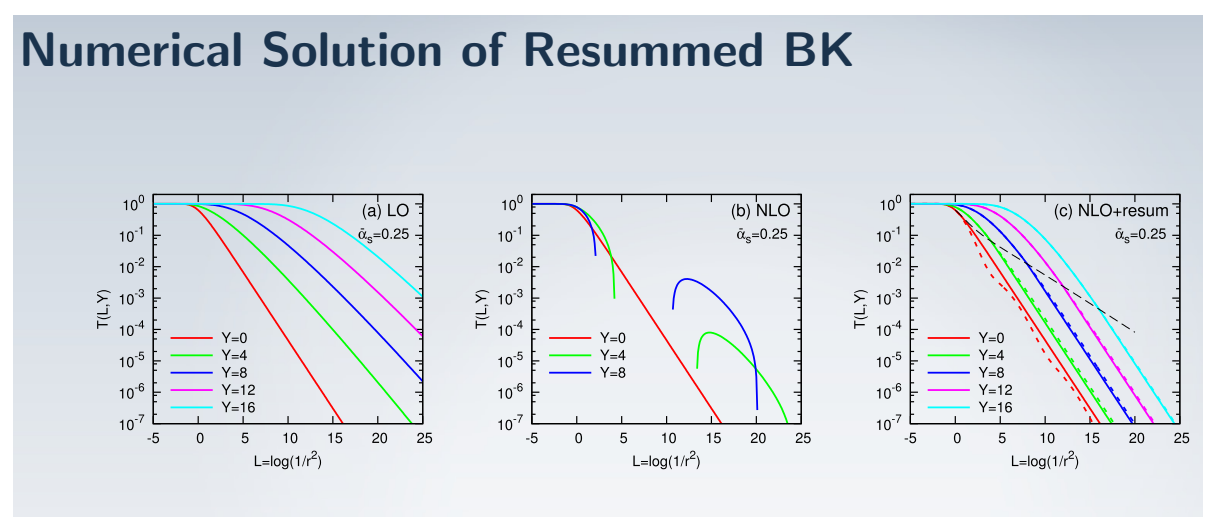

Figure 15: Solution of LO BK (left panel), NLO BK (middle panel) and resummed NLO BK (right panel). Here $T$ is the same as our dipole amplitude $N$.

A possible solution of this conundrum was proposed in the talk of J. Madrigal. The proposal is to resum certain double-logarithmic terms in the BK equation to all orders. The result of such resummation, carried out in [77], is shown in Fig. 15. Clearly the NLO BK solution, depicted in the middle panel of Fig. 15, has problems, which seem to be resolved when one solves the NLO BK equation with the kernel resumming the above-mentioned double-logarithmic contributions. It remains to be seen which conclusions we can draw regarding calculation of higher-order corrections to the non-linear small- $x$ evolution equations and their possible resummation.

Non-perturbative approach to calculating DIS cross sections using AdS/CFT correspondence was presented in the talk of C.-I. Tan [78]. Coulomb corrections to DIS on nuclei were studied in the talk by K. Tuchin [79].

\subsection{Particle production and correlations in pp, pA, AA}

Continuing with the NLO corrections, several talks in WG 2 addressed the NLO corrections to the particle production cross section in the so-called hybrid formalism [80]. The hybrid formalism considers hadron production in a collision of a dilute (not saturated) projectile with a dense (mostly saturated) target, and is perhaps most relevant either to DIS or to $p+A$ collisions. The projectile is described by a PDF in the collinear framework. In the hybrid formalism, a quark or a gluon, described by the PDF of the projectile, scatters on the target as a Wilson line common in the saturation framework. Thus the hybrid formalism mixes collinear factorization with saturation 
physics. The corresponding hadron production cross section can be schematically written as

$$
\frac{d \sigma^{p+A \rightarrow h+X}}{d^{2} p_{T} d y}=f_{a}\left(x, \mu^{2}\right) \otimes N\left(r_{T}, y\right) \otimes H_{a \rightarrow b}\left(r_{T} \mu\right) \otimes D_{h / b}\left(z, \mu^{2}\right),
$$

where $f_{a}$ is the PDF of parton $a, N\left(r_{T}, y\right)$ is the dipole amplitude representing here various correlators of light-cone Wilson lines, $H_{a \rightarrow b}$ is the hard factor representing a parton $a$ turning into parton $b$ while scattering in the target nucleus, and $D_{h / b}$ is the fragmentation function of parton $b$ into the desired harden $h$.
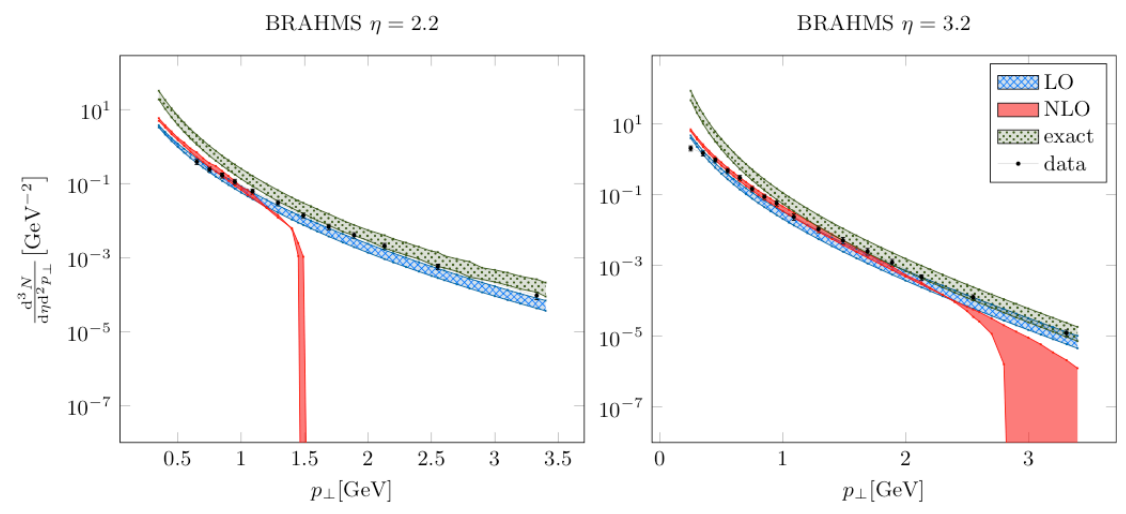

Figure 16: LO and NLO hadron production in the hybrid formalism, along with the exact result in the collinear factorization picture compared to the data reported by the BRAHMS collaboration at RHIC.

NLO corrections to Eq. (3.3) were calculated in [81]. The calculation led to the following problem, discussed in the WG 2 talks by A. Kovner and A. Stasto [82, 83]: the resulting NLO hadron production cross section becomes negative for $p_{T}$ above a certain value. This is illustrated in Fig. 16 showing BRAHMS collaboration data at two different rapidities. Since negative cross section is clearly unphysical, there appears to be a problem in our understanding of particle production at NLO in the saturation framework.

Two proposals on how to deal with the problem were presented in the meeting. Both are related to the fact that one has to make approximations to the exact kinematics in the saturation picture. In the proposal presented by Kovner, a limit is imposed requiring that the coherence length of the parton has to exceed the longitudinal size of the target nucleus. In the talk by Stasto it was argued that at high- $p_{T}$, for $p_{T} \gg Q_{s}$, saturation physics should stop being applicable, mapping onto the collinear factorization expression. The latter correctly includes all the kinematics and leads to strictly positive hadron production cross sections. While both proposal are reasonable and provide a possible way out of the difficult situation, at present it is not quite clear which way of organizing the perturbative expansion is more convenient for the future higher-order calculations.

Particle production and correlations in DIS, $p+p, p+A$ and heavy ion collisions were discussed in the talks by T. Altinoluk [84], B. Gay Ducati [85], A. Dumitru [86], M. Hentschinski [87], P. Kotko [88], A. Rezaeian [89] and D. Wertepny [90].

\subsection{TMDs at small- $x$}

An important new research direction is the convergence of small-x physics and the physics of quark and gluon transverse momentum distributions (TMDs). As was outlined in the talk by 
F. Yuan, the advantage of saturation physics is that it can calculate gluon (and quark) TMDs at low- $x$ more or less from first principles. This can be used to constrain existing and future TMD parametrizations at small- $x$. The suggestion is illustrated in Fig. 17, where the (Fourier transform of the) unpolarized quark TMD is shown calculated in the saturation and collinear approaches.

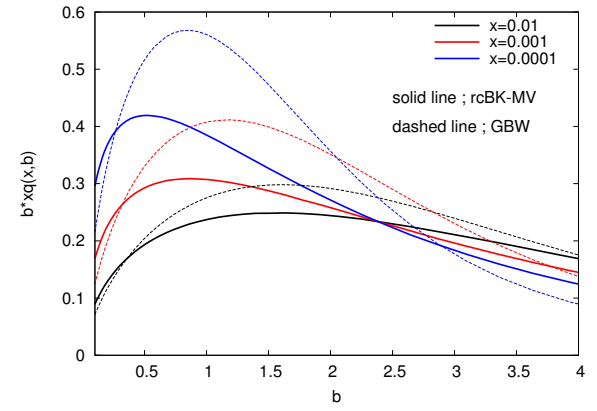

$\mathrm{GeV}^{-1}$

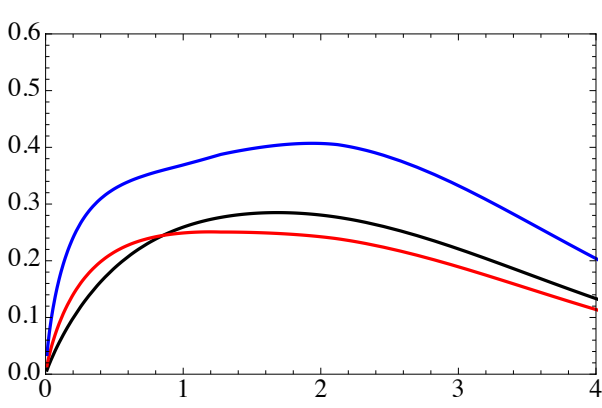

$b, \mathrm{GeV}^{-1}$

Figure 17: Fourier transform into transverse $b_{T}$-space of unpolarized quark TMD calculated in two different saturation-based approaches (left panel) and in the collinear approach (right panel).

In a parallel theoretical development, in the talk by A. Tarasov an evolution equation was presented which maps onto DGLAP, CSS and BK/JIMWLK evolution equations in the appropriate limits [91]. Obtaining all three rather different limits from a single evolution equation could be the first step towards unifying collinear and small- $x$ formalisms.

\section{Acknowledgments}

The material presented in Sec. 3 is based upon work supported by the U.S. Department of Energy, Office of Science, Office of Nuclear Physics under Award Number DE-SC0004286.

\section{References}

[1] L. Adamczyk, Measurement of the total cross section in pp collisions at sqrt(s)=7 TeV with the ALFA sub-detector of ATLAS, these proceedings, PoS(DIS2015)061

[2] G. Aad et al. [ATLAS Collaboration], Nucl. Phys. B 889 (2014) 486

[3] G. Antchev et al. [TOTEM Collaboration], Eur. Phys. Lett. 101 (2013) 21002

[4] G. Antchev et al. [TOTEM Collaboration], Eur. Phys. Lett. 101 (2013) 21004

[5] G. Antchev et al. [TOTEM Collaboration], Eur. Phys. Lett. 101 (2013) 21003

[6] M. Deile, Recent TOTEM Results and Perspectives for the LHC Run 2, these proceedings, PoS(DIS2015) 070

[7] G. Antchev et al. [TOTEM Collaboration], Nucl. Phys. B 899 (2015) 527

[8] G. Antchev et al. [TOTEM Collaboration], Phys. Rev. Lett. 111 (2013) 012001

[9] R. Ciesielski, K. Goulianos, MBR Monte Carlo Simulation in PYTHIA8, [arXiv:1205.1446]

[10] K. Goulianos, Review of RENORM Diffractive Predictions for LHC up to 8 TeV and Extension to 13 $\mathrm{TeV}$, these proceedings, PoS(DIS2015)073 
[11] G. Brona, Diffractive processes in pp collisions at $7 \mathrm{TeV}$ measured with the CMS experiment, these proceedings, PoS(DIS2015)067

[12] V. Khachatryan et al. [CMS Collaboration], Phys. Rev. D 92 (2015) 012003

[13] V. Andreev et. al. [H1 Collaboration], JHEP 03 (2015) 092

[14] S. Schmitt, Measurement of Dijet Production in Diffractive Deep-Inelastic ep Scattering at HERA, these proceedings, PoS(DIS2015)087

[15] S. Schmitt, Diffractive Dijet Production with a Leading Proton in ep Collisions at HERA, these proceedings, PoS(DIS2015)086

[16] V. Andreev et. al. [H1 Collaboration], DESY-14-242, submitted to JHEP

[17] H. Abramowicz et. al. [ZEUS Collaboration], DESY-15-070, submitted to EPJ

[18] L. Adamczyk, Production of exclusive dijets in diffractive deep inelastic scattering at HERA, these proceedings, PoS(DIS2015)062

[19] P. Bussey, Diffractive production of isolated photons at HERA, these proceedings, PoS(DIS2015)068

[20] M. Albrow, The CMS-TOTEM Precision Proton Spectrometers CT-PPS, these proceedings, PoS(DIS2015)064

[21] CT-PPS Technical Design Report, CERN-LHCC-2014-021, CMS-TDR-013, TOTEM-TDR-003 (8 September 2014).

[22] N. Kovalchuk, Measurement of the cross-section ratio $\sigma_{\psi(2 S)} / \sigma_{J / \psi}$ in deep inelastic exclusive ep scattering at HERA, these proceedings, PoS(DIS2015)078

[23] M. Albrow, Measurement of central exclusive pi+pi-production in p-pbar collisions at sort $(s)=0.9$ and $1.96 \mathrm{TeV}$ at CDF, these proceedings, PoS(DIS2015)063

[24] T. Aaltonen et al. [CDF Collaboration], Phys. Rev. D 91 (2015) 091101

[25] R. Wallace, Central exclusive production measurements at $L H C b$, these proceedings, PoS(DIS2015)094

[26] J. Adam, ALICE results on ultra-peripheral $p-P b$ and $P b-P b$ collisions, these proceedings, PoS(DIS2015)060

[27] M. Murray, Ultra peripheral vector meson production from PbPb Collisions at $2.76 \mathrm{TeV}$, these proceedings, PoS(DIS2015)082

[28] R. Aaij et al. [LHCb Collaboration], J. Phys. G: Nucl. Part. Phys. 41 (2014) 055002

[29] R. Aaij et al. [LHCb Collaboration], J. High Energy Phys. 09 (2015) 084

[30] R. Aaij et al. [LHCb Collaboration], J. Phys. G: Nucl. Part. Phys. 41 (2014) 115002

[31] B. Abelev et al. [ALICE Collaboration], PRL 113 (2014) 232504

[32] B. Abelev et al. [ALICE Collaboration], Phys. Lett. B 718 (2013) 1273

[33] B. Abelev et al. [ALICE Collaboration], Eur. Phys. J. C73 (2013) 2617

[34] CMS Collaboration, CMS-PAS-HIN-12-009

[35] B. Abelev et al. [ALICE Collaboration], CERN-PH-EP-2015-082, submitted to JHEP

[36] J. Nystrand, Exclusive and inclusive photonuclear interactions at the LHC, these proceedings, PoS(DIS2015)083 
[37] M.B. Gay Ducati, M. Thurow Griep, M. Machado, Exclusive photoproduction of quarkonium at the LHC energies within the color dipole approach, these proceedings, PoS(DIS2015)072

[38] A. Rezaeian, N. Armesto, Diffractive production from the Color-Glass-Condensate, these proceedings, PoS(DIS2015)085

[39] T. Toll and T. Ullrich, Phys. Rev C87 (2013) 024913

[40] T. Ullrich, Sarte, a generator for diffractive vector meson production in ep and eA collisions, these proceedings, PoS(DIS2015)093

[41] X. Tian, Coherent Rho Meson Production in Neutrino Interaction, these proceedings, PoS(DIS2015)091

[42] J. Asaadi, Detection of CC "O pion" neutrino interactions in LAr TPC with the ArgoNeuT detector, these proceedings, PoS(DIS2015)066

[43] L. V. Gribov, E. M. Levin, and M. G. Ryskin, Semihard Processes in QCD, Phys. Rept. 100 (1983) $1-150$.

[44] I. Balitsky, High-energy QCD and Wilson lines, hep-ph/0101042.

[45] E. Iancu and R. Venugopalan, The color glass condensate and high energy scattering in QCD, hep-ph/0303204.

[46] J. Jalilian-Marian and Y. V. Kovchegov, Saturation physics and deuteron gold collisions at RHIC, Prog. Part. Nucl. Phys. 56 (2006) 104-231, [hep-ph / 0505052 ].

[47] H. Weigert, Evolution at small $x_{b j}$ : The Color Glass Condensate, Prog. Part. Nucl. Phys. 55 (2005) 461-565, [hep-ph/0501087].

[48] F. Gelis, E. Iancu, J. Jalilian-Marian, and R. Venugopalan, The Color Glass Condensate, Ann.Rev.Nucl.Part.Sci. 60 (2010) 463-489, [arXiv: 1002 . 0333].

[49] J. L. Albacete and C. Marquet, Gluon saturation and initial conditions for relativistic heavy ion collisions, Prog.Part.Nucl.Phys. 76 (2014) 1-42, [arXiv:1401.4866].

[50] A. Accardi, J. Albacete, M. Anselmino, N. Armesto, E. Aschenauer, et. al., Electron Ion Collider: The Next QCD Frontier - Understanding the glue that binds us all, arXiv:1212.1701.

[51] Y. V. Kovchegov and E. Levin, Quantum Chromodynamics at High Energy. Cambridge University Press, 2012.

[52] I. Balitsky, Operator expansion for high-energy scattering, Nucl. Phys. B463 (1996) 99-160, [hep-ph/9509348].

[53] I. Balitsky, Factorization and high-energy effective action, Phys. Rev. D60 (1999) 014020 , [hep-ph/9812311].

[54] Y. V. Kovchegov, Small-x $F_{2}$ structure function of a nucleus including multiple pomeron exchanges, Phys. Rev. D60 (1999) 034008, [hep-ph/ 9901281$]$

[55] Y. V. Kovchegov, Unitarization of the BFKL pomeron on a nucleus, Phys. Rev. D61 (2000) 074018, [hep-ph/9905214].

[56] J. Jalilian-Marian, A. Kovner, and H. Weigert, The Wilson renormalization group for low x physics: Gluon evolution at finite parton density, Phys. Rev. D59 (1998) 014015, [hep-ph/ 9709432 ].

[57] J. Jalilian-Marian, A. Kovner, A. Leonidov, and H. Weigert, The Wilson renormalization group for low x physics: Towards the high density regime, Phys. Rev. D59 (1998) 014014, [hep-ph/9706377]. 
[58] E. Iancu, A. Leonidov, and L. D. McLerran, The renormalization group equation for the color glass condensate, Phys. Lett. B510 (2001) 133-144.

[59] E. Iancu, A. Leonidov, and L. D. McLerran, Nonlinear gluon evolution in the color glass condensate. I, Nucl. Phys. A692 (2001) 583-645, [hep-ph/ 0011241$].$

[60] E. A. Kuraev, L. N. Lipatov, and V. S. Fadin, The Pomeranchuk singlularity in non-Abelian gauge theories, Sov. Phys. JETP 45 (1977) 199-204.

[61] I. Balitsky and L. Lipatov, The Pomeranchuk Singularity in Quantum Chromodynamics, Sov.J.Nucl.Phys. 28 (1978) 822-829.

[62] E. Iancu, K. Itakura, and L. McLerran, Geometric scaling above the saturation scale, Nucl. Phys. A708 (2002) 327-352, [hep-ph / 0203137$].$

[63] A. H. Mueller and D. N. Triantafyllopoulos, The energy dependence of the saturation momentum, Nucl. Phys. B640 (2002) 331-350, [hep-ph / 0205167$].$

[64] L. D. McLerran and R. Venugopalan, Green's functions in the color field of a large nucleus, Phys. Rev. D50 (1994) 2225-2233, [hep-ph / 9402335$].$

[65] L. D. McLerran and R. Venugopalan, Gluon distribution functions for very large nuclei at small transverse momentum, Phys. Rev. D49 (1994) 3352-3355, [hep-ph/9311205].

[66] L. D. McLerran and R. Venugopalan, Computing quark and gluon distribution functions for very large nuclei, Phys. Rev. D49 (1994) 2233-2241, [hep-ph/ 9309289 ].

[67] A. M. Stasto, K. Golec-Biernat, and J. Kwiecinski, Geometric scaling for the total $\gamma^{*} p$ cross-section in the low x region, Phys. Rev. Lett. 86 (2001) 596-599, [hep-ph/ 0007192$].$

[68] M. Praszalowicz and A. Francuz, Geometrical Scaling in Inelastic Inclusive Particle Production at the LHC, arXiv: 1507.0818.

[69] ALICE Collaboration, B. B. Abelev et. al., Energy Dependence of the Transverse Momentum Distributions of Charged Particles in pp Collisions Measured by ALICE, Eur. Phys. J. C73 (2013), no. 12 2662, [arXiv:1307.1093].

[70] V. S. Fadin and L. N. Lipatov, BFKL pomeron in the next-to-leading approximation, Phys. Lett. B429 (1998) 127-134, [hep-ph/9802290].

[71] M. Ciafaloni and G. Camici, Energy scale(s) and next-to-leading BFKL equation, Phys. Lett. B430 (1998) 349-354, [hep-ph / 9803389$]$.

[72] I. Balitsky and G. A. Chirilli, Next-to-leading order evolution of color dipoles, Phys.Rev. D77 (2008) 014019, [arXiv:0710.4330].

[73] I. Balitsky and G. A. Chirilli, Rapidity evolution of Wilson lines at the next-to-leading order, Phys.Rev. D88 (2013) 111501, [arXiv: 1309.7644 ].

[74] A. V. Grabovsky, Connected contribution to the kernel of the evolution equation for 3-quark Wilson loop operator, JHEP 09 (2013) 141, [arXiv: 1307. 5414].

[75] A. Kovner, M. Lublinsky, and Y. Mulian, Jalilian-Marian, Iancu, McLerran, Weigert, Leonidov, Kovner evolution at next to leading order, Phys. Rev. D89 (2014), no. 6061704 , [arXiv:1310.0378].

[76] T. Lappi and H. Mantysaari, Direct numerical solution of the coordinate space Balitsky-Kovchegov equation at next to leading order, Phys. Rev. D91 (2015), no. 7 074016, [arXiv: 1502.0240 ]. 
[77] E. Iancu, J. D. Madrigal, A. H. Mueller, G. Soyez, and D. N. Triantafyllopoulos, Resumming double logarithms in the QCD evolution of color dipoles, Phys. Lett. B744 (2015) 293-302, [arXiv:1502.0564].

[78] R. C. Brower, M. Djuric, I. Sarcevic, and C.-I. Tan, String-Gauge Dual Description of Deep Inelastic Scattering at Small-x, JHEP 11 (2010) 051, [arXiv: 1007.2259 ].

[79] K. Tuchin, Coulomb corrections to photon and dilepton production in high energy pA collisions, Phys. Rev. C89 (2014), no. 2 024904, [arXiv:1311.1124].

[80] A. Dumitru and J. Jalilian-Marian, Forward quark jets from protons shattering the colored glass, Phys. Rev. Lett. 89 (2002) 022301, [hep-ph/ 0204028 ].

[81] G. A. Chirilli, B.-W. Xiao, and F. Yuan, Inclusive Hadron Productions in pA Collisions, Phys. Rev. D86 (2012) 054005, [arXiv:1203.6139].

[82] T. Altinoluk, N. Armesto, G. Beuf, A. Kovner, and M. Lublinsky, Single-inclusive particle production in proton-nucleus collisions at next-to-leading order in the hybrid formalism, Phys. Rev. D91 (2015), no. 9 094016, [arXiv:1411.2869].

[83] A. M. Stasto, B.-W. Xiao, F. Yuan, and D. Zaslavsky, Matching collinear and small x factorization calculations for inclusive hadron production in pA collisions, Phys. Rev. D90 (2014), no. 1014047 , [arXiv:1405.6311].

[84] T. Altinoluk, N. Armesto, G. Beuf, M. Martinez, and C. A. Salgado, Next-to-eikonal corrections in the CGC: gluon production and spin asymmetries in pA collisions, JHEP 07 (2014) 068, [arXiv:1404.2219].

[85] M. B. Gay Ducati, M. T. Griep, and M. V. T. Machado, Exclusive photoproduction of J/psi and psi(2S) states in proton-proton collisions at the CERN LHC, Phys. Rev. D88 (2013) 017504, [arXiv:1305.4611].

[86] A. Dumitru and V. Skokov, Anisotropy of the semiclassical gluon field of a large nucleus at high energy, Phys. Rev. D91 (2015), no. 7 074006, [arXiv:1411.6630].

[87] M. Hentschinski, J. D. M. Martinez, B. Murdaca, and A. S. Vera, The Gluon-Induced Mueller-Tang Jet Impact Factor at Next-to-Leading Order, Nucl. Phys. B889 (2014) 549-579, [arXiv:1409.6704].

[88] P. Kotko, K. Kutak, C. Marquet, E. Petreska, S. Sapeta, and A. van Hameren, Improved TMD factorization for forward dijet production in dilute-dense hadronic collisions, arXiv: 1503.0342.

[89] N. Armesto and A. H. Rezaeian, Exclusive vector meson production at high energies and gluon saturation, Phys. Rev. D90 (2014), no. 5 054003, [arXiv: 1402 . 4831].

[90] G. A. Chirilli, Y. V. Kovchegov, and D. E. Wertepny, Classical Gluon Production Amplitude for Nucleus-Nucleus Collisions: First Saturation Correction in the Projectile, JHEP 03 (2015) 015, [arXiv:1501.0310].

[91] I. Balitsky and A. Tarasov, Rapidity evolution of gluon TMD from low to moderate $x$, arXiv:1505.0215. 